\title{
Clinical case of the chicken pox complicated with meningoencephalitis
}

\section{Opinion}

Registered annually 80-90 million cases of chickenpox in the world. Chickenpox is a highly contagious exanthematous infection, ending at $95 \%$ recovery, with a maximum incidence between the ages of 3 and 7 years. The remaining 5\% of disease has complications in immunocompromised individuals incidence of complications increases and reaches $30 \%-50 \%$. In 1 out of 50 cases observed as a complication of pneumonia, which can be either primary viral and bacterial origin. The most formidable complications include development of encephalitis which is detected in 1 of 4000 cases of chickenpox. Nerve damage varicella most often occurs on the 5-10 day of illness. They are more common in boys than in girls, between the ages of 1 and 5 years. On encephalitis accounts for $0,1-0,2 \%$ of all patients with varicella and $13 \%$ of all encephalitis established etiology. $75 \%$ encephalitis varicella occur with lesions of the cerebellum and manifest cerebellar ataxia. The clinical picture in these growing for 3-5 days and lasts an average of 2-4 weeks. In 25\% of encephalitis varicella there is an inflammatory process involving the other structures of the brain (brain, brainstem, basal ganglia, and others.). With these forms of encephalitis mortality may reach $35 \%$, and the residual effects in the form of paralysis, mental retardation, repeated seizures may occur in $12 \%-15 \%$ of patients. an average of $2-4$ weeks. In $25 \%$ of encephalitis varicella there is an inflammatory process involving the other structures of the brain (brain, brainstem, basal ganglia, and others.). With these forms of encephalitis mortality may reach $35 \%$, and the residual effects in the form of paralysis, mental retardation, repeated seizures may occur in $12 \%-15 \%$ of patients. an average of 2-4 weeks. In $25 \%$ of encephalitis varicella there is an inflammatory process involving the other structures of the brain (brain, brainstem, basal ganglia, and others.). With these forms of encephalitis mortality may reach $35 \%$, and the residual effects in the form of paralysis, mental retardation, repeated seizures may occur in $12 \%-15 \%$ of patients.

Mortality varicella among children aged 1-14 years in the world is about 2 cases per 100,000. Despite these facts to the public, including medical clinics, rooted opinion that chickenpox is always favorable course. The result of this error is not timely diagnosis of complications, leading to untimely and inadequate therapy and as a result of possible disability or death of the patient. We observed K. girl, 5 years old, who attend kindergarten, with the words of my mother - in group and family cases of chickenpox were reported. Life history unremarkable, except for clinical supervision by a neurologist before 1 year of age with a diagnosis of perinatal lesions of the central nervous system, which also may be a risk factor for the development of complicated chickenpox. From anamnesis it is known that the first complaints of rashes all over the skin surface were 26.11., Was treated under the supervision of a dynamic local pediatrician local tushirovanie elements rash alcoholic solution of brilliant green and antihistamines in age dosage inside. rashes period lasted 5 days, fever is not mentioned, the girl did not suffer health. After 2 days from the last lesions or 7 days from the beginning of the disease there were complaints on lethargy, dizziness and nausea, even the next day the child refused to sit on the bed, with a change in body position was observed vomiting not associated with
Volume 5 Issue 2 - 2018

\author{
Berezovskaya Tatiana S, 1,2 Miromanova \\ Natalia A, ${ }^{1,2}$ Zvereva Larisa Alekseevna, ${ }^{2}$ \\ Feminine Ekaterina, ${ }^{2}$ Miromanov Alexander' \\ 'Chita State Medical Academy, Russia \\ ${ }^{2}$ Regional Clinical Hospital of Infectious Diseases, Russia
}

Correspondence: Tatiana S Berezovskaya, Regional Clinical Hospital of Infectious Diseases, Chita Street. Bitter, 39a FGBOU IN Chita State Medical Academy, Russia, Tel 8 (3022) 39-5I-48, Email baranchygavats@mail.ru

Received: August 28, 20I8 | Published: November 15, 2018

food intake, appeared unsteady gait. Fever was observed. The girl examined by the district pediatrician diagnosis remained the same, but is connected to the therapy of interferon preparation is $a-2 b$ inside. The next day marked drowsiness, complaints of nausea and headache, failure to sit and walk. Mom recall a pediatrician, and after examining the girl was sent to the regional clinical infectious diseases hospital with the diagnosis: Varicella. Meningitis? with a change in body position was observed vomiting not associated with food intake, it appeared unsteady gait. Fever was observed. The girl examined by the district pediatrician diagnosis remained the same, but is connected to the therapy of interferon preparation is $a-2 b$ inside. The next day marked drowsiness, complaints of nausea and headache, failure to sit and walk. Mom recall a pediatrician, and after examining the girl was sent to the regional clinical infectious diseases hospital with the diagnosis: Varicella. Meningitis? with a change in body position was observed vomiting not associated with food intake, it appeared unsteady gait. Fever was observed. The girl examined by the district pediatrician diagnosis remained the same, but is connected to the therapy of interferon preparation is $a-2 b$ inside. The next day marked drowsiness, complaints of nausea and headache, failure to sit and walk. Mom recall a pediatrician, and after examining the girl was sent to the regional clinical infectious diseases hospital with the diagnosis: Varicella. Meningitis? Mom recall a pediatrician, and after examining the girl was sent to the regional clinical infectious diseases hospital with the diagnosis: Varicella. Meningitis? Mom recall a pediatrician, and after examining the girl was sent to the regional clinical infectious diseases hospital with the diagnosis: Varicella. Meningitis? On admission to the hospital complaint expressed weakness, headache pain without clear localization, nausea and vomiting with a change of position of the body, dizziness, shaky and unsteady gait. The patient's condition serious due to neurological symptoms: marked a stunning gipotopiya muscles of the right upper extremity, positive Babinski left, stiff neck muscles to 2 centimeters, positive symptom Kernig on both sides, with the finger - nose test indicated mimopopadanie, heel - knee samples, the uncertain. On the skin crisp tushirovat green diamond, no fever, lung auscultation vesicular respiration, tendency to arterial hypotension, and other indicators within the age norm. Hospitalized in the intensive care unit. On the day of arrival counseled neurologist and ophthalmologist, clinical diagnosis: Varicella. Secondary infection allergic meningoencephalitis. swelling of the brain. Ataxic syndrome. 
The therapy specific antiviral and antibacterial drugs, hormone therapy, disintoxication and anti-edema therapy, neuroprotective and vitamin in accordance with age dosages.

In general blood test for admission without pathology, after 5 days - neutrophilic hyperskeocytosis (clinically accompanied by a rise in body temperature without the addition of secondary flora). In biochemical analysis a blood boundary acute phase component of inflammation $\mathrm{C}$ - reactive protein is $6.0 \mathrm{mg} / 1$ - no dynamics in the control. By polymerase chain reaction of blood and content elements rash isolated DNA virus Varicella Zoster. Cerebro - spinal puncture is not carried out, since during the spinous processes of the vertebrae in the lumbar region marked thickening of residue elements rash. On day 4 from virginsPoints marked increase in body temperature to febrile digits - increased dose antibiotic therapy and connected immunovenin. Re-examined by a neurologist - a result of the choice of a comfortable position the baby's head to reduce nausea and refusal to sit down and go, marked hypertonus sterno - clavicular - mastoid muscles of the right and left rotation of the foot. The recommendations on the need of the therapeutic loading and carrying a light massage the muscles of certain groups. Against the backdrop of ongoing remedial measures and through hard comply with all recommendations mother child neurologist at the start of rehabilitation, to 11 day from the date of admission of the girl were minor phenomenon asthenia, and ataxia.
For further treatment and rehabilitation transferred to the Department of Neurology Regional Children's Hospital.

Danny clinical case indicates the formation of a relatively favorable outcome of the disease with the possible formation of minimal brain dysfunction, despite the late diagnosis of clinical complications and the lack of timely specific treatment. butIt should be remembered that every case of chicken pox, the patient may have a potentially adverse outcome. The authors note that despite the fact the costeffective course of chickenpox, across the state, is a natural process of formation of immunity after the disease. But given that all cases of reflow of varicella in adults more frequently recorded, which has a heavy and complicated course, as well as the development of complications in children requires a mass immunization by administration of the vaccine in the national calendar of preventive vaccinations in all regions of the country. Reaching the maximum number of children's vaccine prophylaxis of varicella in avoiding death and disability in the population.

\section{Acknowledgements}

None.

\section{Conflict of interest}

The authors declare that there is no conflict of interest. 